\title{
Una splendida cittadella fortificata a presidio dello Stretto di Messina
}

\author{
Massimo Lo Curzioa , Marco Lo Curzio \\ ${ }^{a}$ Università Mediterranea_Master EMDiReB, Reggio Calabria, Italy, mlocurzio@gmail.com, b Accademia di Belle \\ Arti, L'Aquila, Italy, marco.locurzio@gmail.com.
}

\begin{abstract}
The Real Citadel of Messina is among the most spectacular fortifications built in the Mediterranean in the late seventeenth century. Planned by the Spaniard rulers following the bloody suppression of the 1674-78's revolt, Carlos de Grunenbergh conceived it according to two converging needs: controlling the Strait of Messina and inhibiting future ideas of revolt. Significant presence with a strong visual impact.

Set in the middle of the "scythe" of the natural harbour, this imposing appearance results from a well definite pentagonal layout. Conceived as a "citadel", unlike the other fortifications of the city laid out as "strongholds" on the hills, it has a considerable size and the possibility of military hosting. Today it lays in poor condition, partly destroyed, badly preserved and unfitted to a correct use. A program of actions would be advisable for its conservation in order to give the monument a new life, within an innovative circuit of functions.
\end{abstract}

Keywords: total approach, reuse strategy, restoration, Strait of Messina.

\section{Introduzione}

Da un lato si manifesta drammaticamente la consueta considerazione generale: come conservare una struttura nata per la guerra e aborrita da chi ne ha visto la manifestazione come un sopruso; dall'altra resta l'impianto e l'impronta di un bene assolutamente particolare che reclama un possibile restauro. Recupero di spazi, di ricchezza d'impianto, di soluzioni compatibili e di prefigurazioni possibili. Conservazione di un bene che è stato sezionato, parzialmente distrutto per fare una stazione che è un capolavoro dell'architettura futurista italiana, mutilato per presunti scopi produttivi dell'area.

Indissolubili sono quindi il restauro, la conservazione dell'esistente e la capacità - o volontà - di vivere in maniera consapevole e contemporanea un impianto superbo posto in un contesto ambientale spettacolare. Nel 2003, con un progetto redatto con la Soprintendenza ai beni culturali per la creazione di un centro di documentazione per le arti contemporanee, si è avviato un programma di conservazione e riutilizzo e abbiamo messo a punto un'ipotesi di intervento. Obiettivi fondamentali: il restauro delle parti in crisi e una destinazione d'uso per servizi culturali. Ma il finanziamento, malgrado il progetto approvato, è stato perso per "disattenzione politica". Nel 2013 con l'occasione di un Master internazionale denominato EMDiReB (European Master in Diagnosis and Repair of Buildings) del programma UE Erasmus Mundus riservato ad ingegneri e architetti di paesi terzi e UE, specialisti in interventi sul patrimonio culturale, sono state formulate importanti proposte di restauro e riqualificazione dell'intera area che si sono confrontate con la dimensione concettuale 
dei MasterPlan e con previsioni di ridefinizione formale che affrontano, in un approccio totale al progetto il restauro della fabbrica, la questione del recupero dell'immagine rispetto a quanto è stato perso nel tempo e alla relazione con l'immagine della città*.

Il prodotto di queste iniziative, espresso da diverse formulazioni progettuali, è frutto di stimolanti approcci al restauro e al dibattito sulla conservazione che, relativamente all'impianto generale, è doverosamente partito dal desiderio di praticare i principi della carta di Amsterdam e di trovare soluzioni strategicamente vincenti.

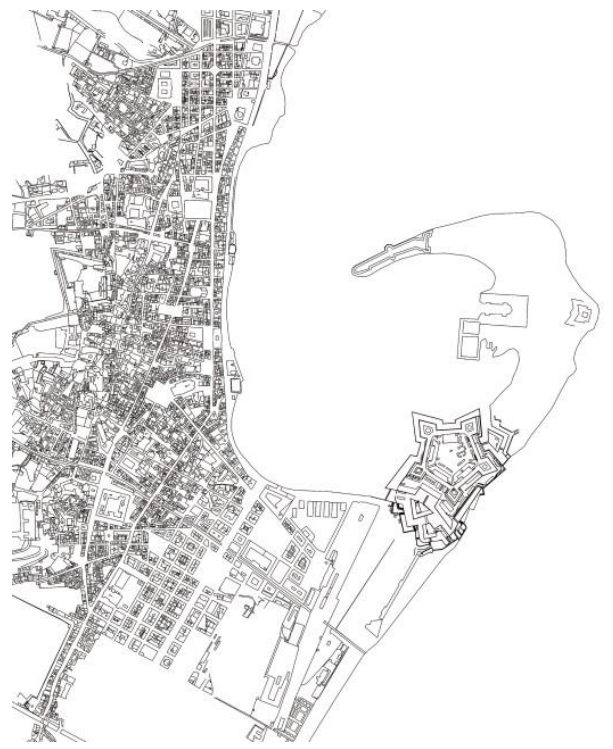

Fig. 1- La città di Messina nell'Ottocento. Si noti la relazione tra il tessuto urbano e la Cittadella.

\section{La forma e il contesto}

Tutte le formulazioni messe a punto partivano, necessariamente, dalla necessità di ricomposizione della forma d'impianto come matrice di una nuova organizzazione in grado di dare forza e coerenza ad una posizione precisa e vincente. Il perfetto pentagono realizzato dal De Grunenberg è figura simbolica che vuole avere come riferimento lo Stretto, come luogo di interesse fondamentale e la città come ribelle presidio umano frontistante. Il modello architettonico è di complessa interpretazione. Da un lato parte dalle sperimentazioni rinascimentali che ci fanno pensare ad esempio a Leonardo per l'impianto degli indispensabili rivellini, dall'altro è figlio del dibattito che attraversa il XVII secolo e che vede nelle sperimentazioni del Vauban i livelli di elaborazione più alta.

La formazione del De Grunenberg sembra assecondare quest'ipotesi che qui si riporta sinteticamente. Certo è che l'impianto della Cittadella corrisponde ad una delle realizzazioni più lucidamente estreme che il periodo consente. La splendida falce del porto di Messina viene sezionata sulla base di un disegno assai preciso e allagata per stabilire una cesura con la città ribelle. Lo stesso vale per il fronte rivolto a nord, luogo delle precedenti strutture militari. La Cittadella è e vuole essere un'isola imprendibile, in grado di ospitare un rilevante numero di occupanti stabili e pronta a moltiplicare le sue possibilità offensive. Questa matrice progettuale la rende contemporaneamente di felice impianto geometrico e occasione di assoluto distacco dalla città storica. Esemplare e perfetta da un lato, assolutamente scissa dalla vita reale del contesto dall'altro.

Evidente quindi che connessione, o meglio il dialogo, tra la città e la Cittadella va impostato come nuova e complessa rivisitazione delle relazioni che va cablata con i necessari livelli progettuali.

Di fatto alla base del discorso sta l'impianto di uno strumento di messa a punto del funzionamento dell'intera area che abbiamo definito con l'uso del Masterplan, disegno generale e prefigurazione materiale di organizzazione delle funzioni ammissibili secondo una necessaria visione strategica avanzata e vincente.

\section{L'immaginario urbano: un approccio al restauro totale}

Sosteniamo per un bene di questa consistenza, per il valore storico e la relazione con il contesto urbano, un approccio al restauro di tipo "totale". Totale nel senso metodologico, inteso cioè a ristabilire le qualità dei manufatti valorizzando le permanenze architettoniche ma considerando un più ampio approccio alle valenze formali del 
progetto originario in termini di forma percepita, storicizzata e appartenente alle nuove configurazioni dell'immaginario urbano

L'immagine della città e quella persistenza percettiva che si determina nei suoi spazi costruiti e relazionati permettendone la lettura, l'orientamento e il ricordo nei suoi utenti/abitanti. Molti studi ne hanno tracciato la validità metodologica nei processi di ridisegno urbano, e in particolare le teorie di Kevin Lynch alla fine degli anni '60 ne hanno sancito le basi per un uso strutturato nella prassi urbanistica. Santificate dai teorici ma mai del tutto abbracciate dai tecnici, queste ricerche sono state criticate, soprattutto in Europa per un presunto eccesso di pragmatismo ed alcune ingenuità storicistiche, ma tornano oggi a sembrare valide intuizioni anche alla luce dell'inadeguatezza dei modelli strategici pianificatori che hanno determinato il collasso dell'identità della città contemporanea.

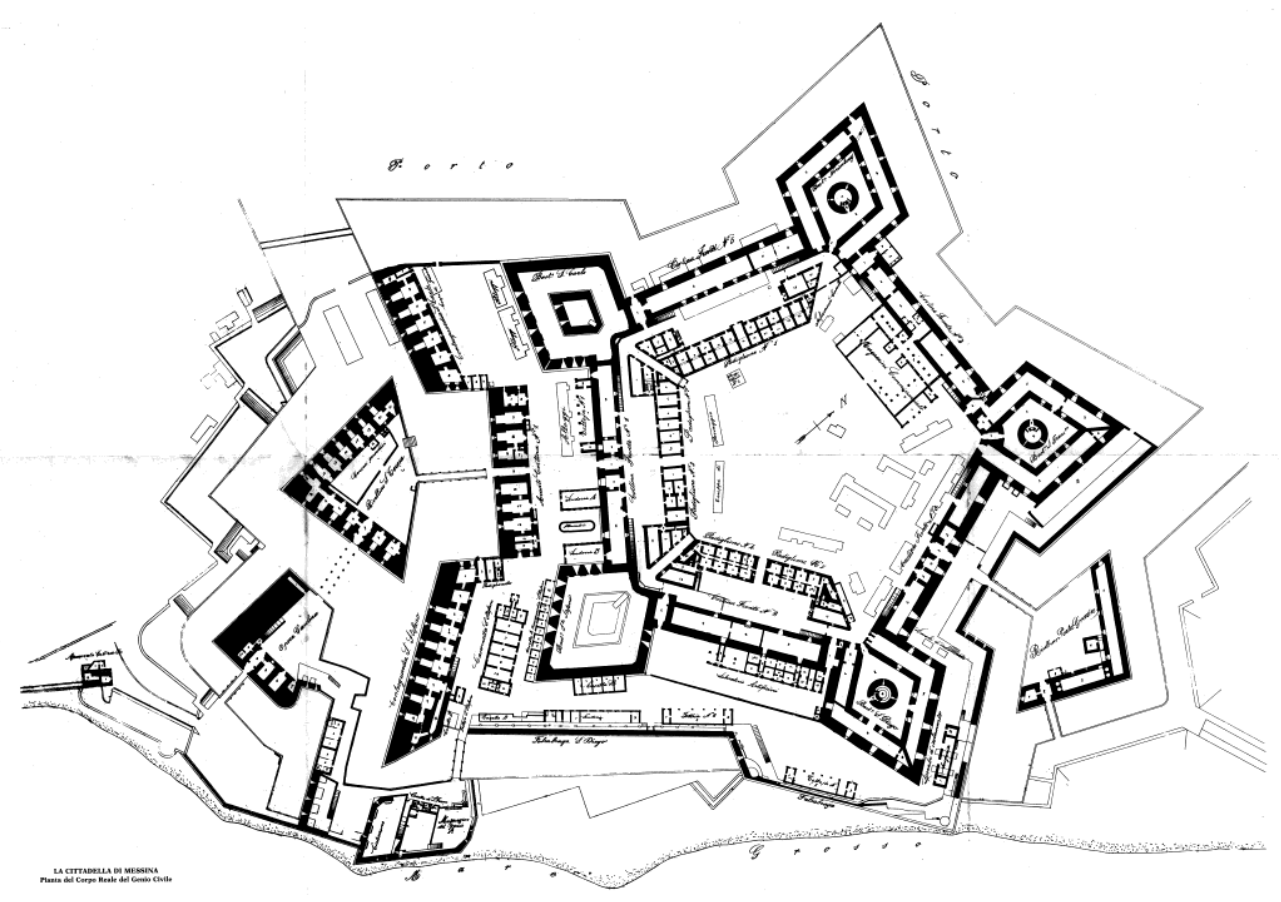

Fig. 2- La Real Cittadella di Messina nel 1885. Rilievo del Genio Civile

D'altra parte Lynch confinava il suo lavoro dentro le questioni più rilevanti delle città statunitensi, la cui crescita rapida ed eterogenea in quegli anni, anticipava problemi di smarrimento del "senso" dell'abitare, portando al fianco degli architetti, sociologi e psicologi e ponendo problemi di ecologia dell'immaginario.

Il problema della lettura dello spazio a partire dalla percezione dei suoi utenti, in realtà afferma l'esigenza metodologica di porsi in "ascolto" della forma urbana, e delle relazioni reciproche di comprensibilità tra questa e i suoi abitanti, del suo potenziale vivibile dunque, ma anche della sua potenza vissuta; un atteggiamento secondo cui la storia ridiventa valore percepibile di consapevolezza, e quindi identità, dell'immagine urbana.

Una prospettiva del genere affronta molte delle parole chiave del dibattito contemporaneo sulla città, l'identità multiculturale, la centralizzazione delle periferie, i vuoti urbani post-industriali, i non-luoghi, la stratificazione semantica dei sistemi informativi. 
Lynch suggerisce concetti come progettazione partecipata e sostenibilità ambientale che appartengono alle strategie contemporanee di argine alla spersonalizzazione della città, a cui non sono immuni i centri storici, pericolosamente in sospeso tra il rischio di museificazione, e quindi isolamento, e quello di gentrificazione ovvero spersonalizzazione.

Il complesso della Cittadella di Messina, è un oggetto urbano particolarmente significativo da affrontare in un'ottica di recupero "totale". Se all'evidenza formale in quanto straordinaria testimonianza storica di architettura militare e tecnica costruttiva, affianchiamo la lettura del suo valore "figurale" all'interno della forma della città e delle sue modificazioni, troviamo un patrimonio di enorme valore culturale ma capace anche di un ruolo centrale come oggetto di una strategia attiva di ricostruzione della percezione e della fruizione di una porzione consistente di intorno urbano e, per indotto, dell'intera città. Non si tratta solo di attivare un pensiero conservativo o volumetrico, o strumentale nel recupero dell'architettura storica, ma il contributo potenziale come parte di un "ritratto urbano" che è un sistema di segni sensibili e comprensibili, fulcro di azioni di pianificazioni più complesse.

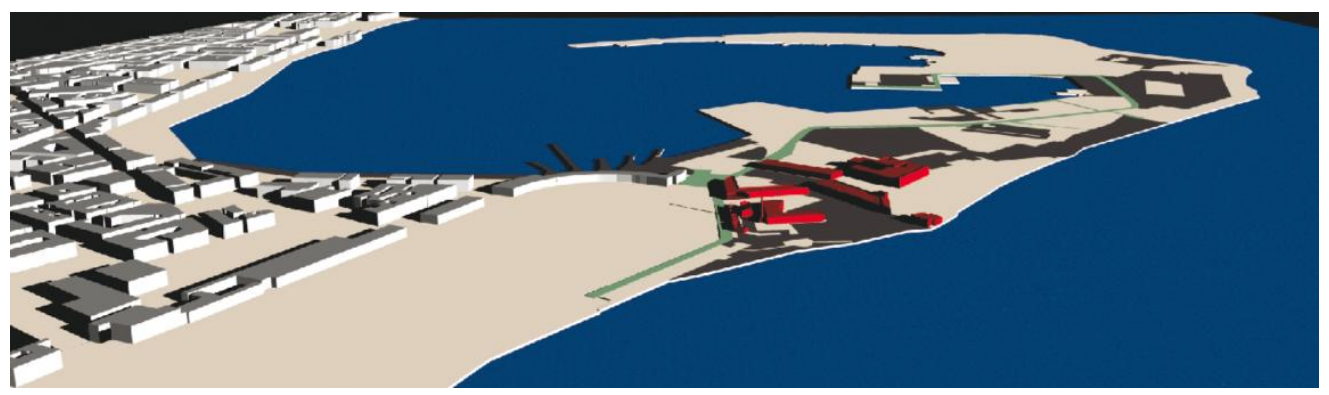

Fig. 3- Progetto Cdac, 2003. Localizzazione dei resti della Cittadella in rosso
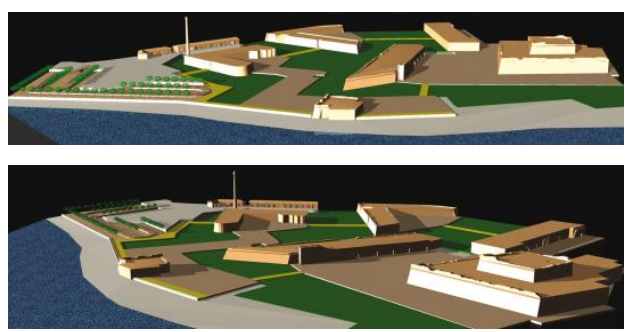

Fig. 4 e 5- Progetto Cdac, 2003. Recupero dell'area e organizzazione volumi esistenti

In fondo anche originariamente il grande pentagramma del progetto del Carlos de Grünenbergh, come già citato, non è funzionale al solo disegno difensivo, ma è configurato come elemento "segnico" di valore simbolico, di identità forte, microcosmo isolato e blindato in ogni direzione e posizionato in relazione allo spazio urbano per essere percepito come interruzione, cesura violenta di un percorso di confine (presidio del limite) e quindi punto di riferimento fortificato, ostentazione di potenza, segno inequivocabilmente dominante.

Non è difficile pensare che fosse nelle intenzioni progettuali originarie proprio questo atteggiamento privativo di una continuità di percorso (libertà) con una struttura di rilevante imponenza, visibilità e leggibilità (segnale), ben oltre quanto fosse necessario per il raggiungimento del solo scopo difensivo. È forse non è un caso che questa originaria cesura abbia innescato, con la concomitanza di molti altri fattori politici, un lento e inesorabile degrado dell'area, relegata a anacronistico baluardo difensivo prima, e successivamente a risorsa industriale presto abbandonata, ma sempre in un ottica percettiva di negazione di una libertà di circolazione e fruizione. 


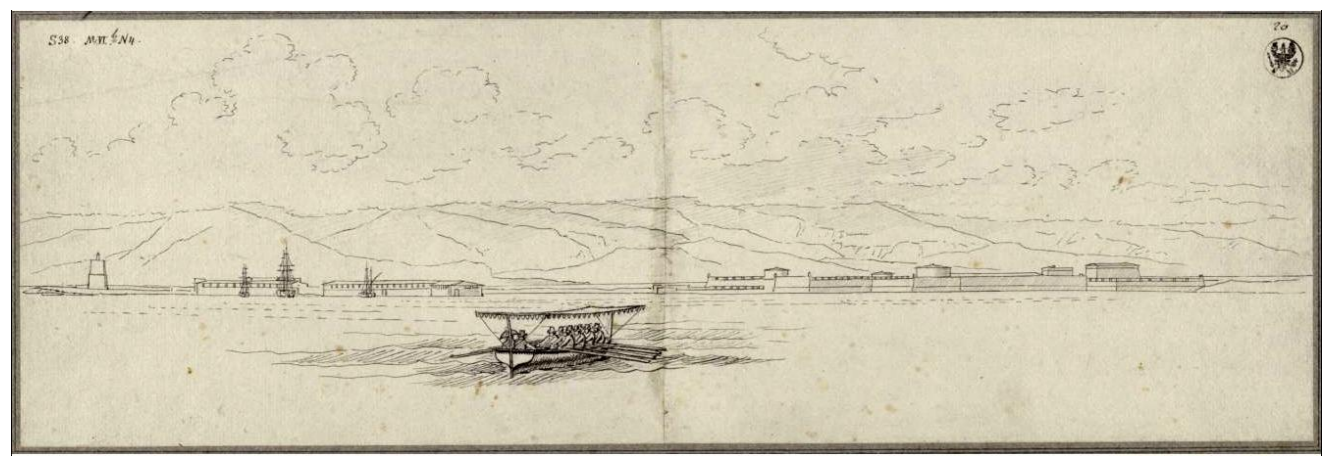

Fig. 6- 1804 - Karl Friedrich Schinkel, Vista dal centro del porto verso la Cittadella, a destra

\section{Riflessioni conclusive}

Leggibilità e figurabilità del segno urbano nella Cittadella di Messina (e in molto del patrimonio fortificato mediterraneo) sono dunque valori di progetto, da recuperare nelle valenze percettive che possono rappresentare nei confronti delle continue conformazioni dell'immagine urbana.
Il bene ha perso nella storia buona parte della consistenza dei manufatti e ogni scopo difensivo, ma conserva un potenziale simbolico formalmente ancora percepibile ed evidenziabile in relazione alla città proprio perché nato dal rapporto di antagonismo con questa che va decisamente ribaltato.

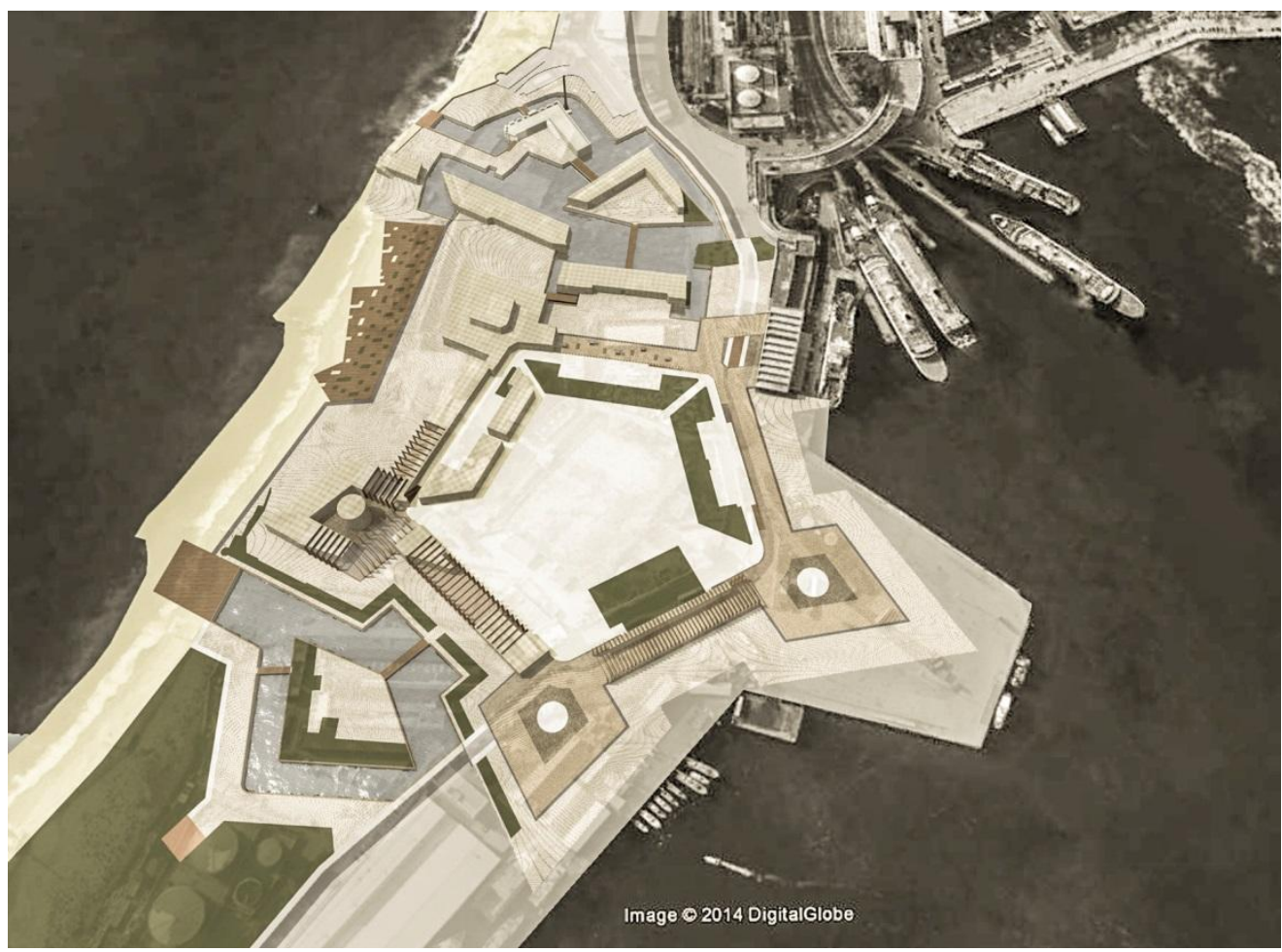

Fig. 7 - Master EMDiReB, Gruppo Carditello-Guerra-Torres. La restituzione della forma della Cittadella nel contesto urbano. 


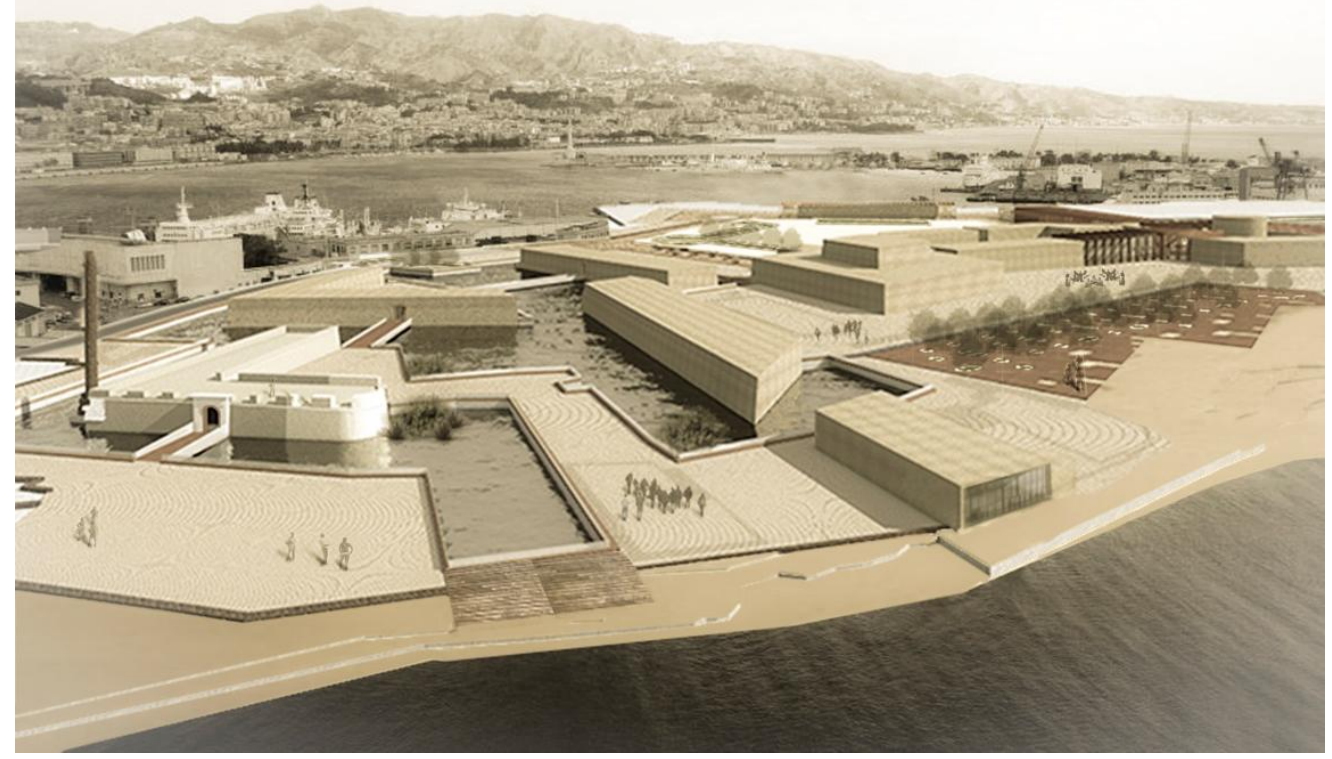

Fig. 8 - Master EMDiReB, Gruppo Carditello-Guerra-Torres. Progetto della Cittadella, render lato sud

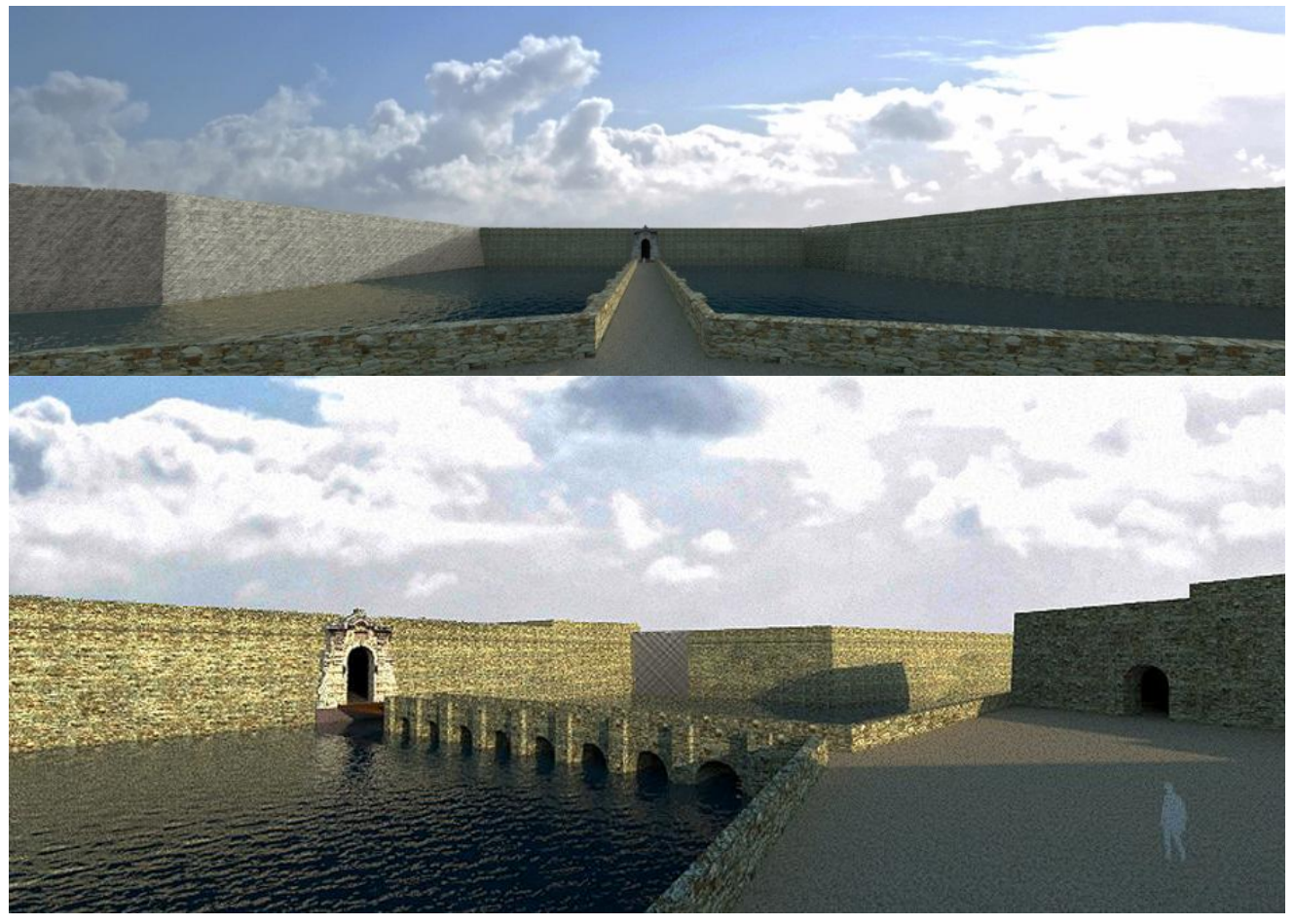

Fig. 9 e 10 - Master EMDiReB, Gruppo Dunn-Gaitan-Vera. Progetto Cittadella, recupero dei fossati allagati. 


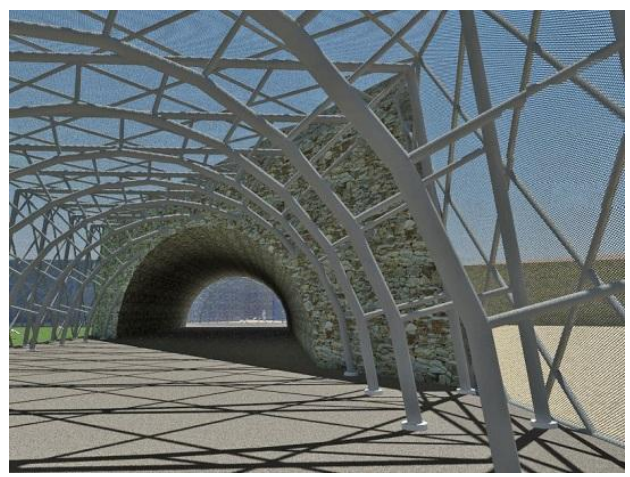

Fig. 11 - Master EMDiReB, Gruppo Dunn-GaitanVera. Progetto Cittadella, continuità degli spazi e nuove strutture.

Il patrimonio fortificato del Mediterraneo è, per gran parte, oggi una straordinaria occasione per riflettere sull'evoluzione della percezione dell'immaginario urbano delle nostre città; una forma di restauro che estenda l'approccio filologico alla fabbrica, arricchendolo dei valori relazionali e percettivi delle trasformazioni in atto nelle città contemporanee potrebbe essere il valore aggiunto nei processi di riqualificazione dei nostri spazi collettivi di matrice storica. In questa direzione sono stati gestiti i progetti per la
Cittadella di Messina svolti nell'ambito del Master EMDIREB, tutti caratterizzati da un approccio al recupero delle modeste evidenze architettoniche nel contesto delle valenze formali del disegno originario complessivo, trattato come snodo e riferimento di valore simbolico e percettivo, nel recupero urbano dell'intera area auspicato nelle politiche di trasformazione della città futura.

\section{Note}

* Gli architetti e ingegneri studenti del Master EMDiReB che hanno presentato i progetti al Comune di Messina, su iniziativa congiunta della direzione del Master, del Sindaco di Messina e del Soprintendente ai Beni Culturali e Ambientali della Provincia di Messina, il 20 febbraio 2014, sono: Yadira Ofarril Rodriguez (Cuba), Emilio Gaston Polo Friz (Argentina), Paula Patricia Almonacid Marquez (Colombia), Jorge Alfredo Gaitan Barrios (Guatemala), Florencia Mariana Mantero (Argentina), Francesca Carditello (Italia), Andrea Guerra Miguez (Spagna), Isabel Vera Franco (Spagna), Christian Maxwell Dunn (Usa) e Veronica Torres de Sande (Spagna).

\section{References}

Aricò Nicola (2002). Segni di Gea, grafie di Atlante. In DRP_Dipartimento di Rappresentazione e Progetto dell'Università di Messina, Rassegna di Studi e Ricerche a cura di Nicola Aricò, N.4-2002. pp. 19-88.

Aricò Nicola (2008). Carlos de Grunenbergh e le città ioniche del Teatro Geografico Antiguo y Moderno del Reyno de Sicilia (1686), «Lexicon» n. 7/2008.

Ioli Gigante Amelia (2010). Messina. Storia della città tra processi urbani e materiali iconografici, a cura di Giovanni Molonia. Libreria Ciofalo.

Lo Curzio M., Caruso V. (2006). La fortificazione permanente dello Stretto di Messina, Storia, conservazione e restauro di un patrimonio architettonico e ambientale. EDAS ed. Messina.

Lo Curzio Massimo (2000). Historical Architectures, urban retrainings and mantenance of a patrimony at risk in Italy in Reconstruction and the politics of space (Atti $7^{\circ}$ Conferenza IASTE, Center for Environmental Design Research, The end of Tradition?), Volume 131, IASTE Working Paper Series, University of California, Berkeley.

Lo Curzio Massimo (2010). Cultural heritage and fortification built for defence of the Strait of Messina in the late $19^{\text {th }}$ century, in Bodownictwo i Architektura, vol. 7 (2) 2010, Politecnika Lubelscka, Lublin (PL).

Lynch Kevin (2006 - 1964). L'immagine della città. Marsilio ed. Venezia.

Lynch Kevin (1990). Progettare la città, la qualità della forma urbana. ETAS ed. Milano. 
Manganaro Mario (2002). Progressivi avvicinamenti al luogo. In DRP_Dipartimento di Rappresentazione e Progetto dell'Università di Messina, Rassegna di Studi e Ricerche a cura di Nicola Aricò, N.4-2002. pp. 89-117.

Riccobono F., Adolfo Berdar A., La Fauci C. (1988) La Real Cittadella di Messina, prefazione di Rodo Santoro. EDAS ed. Messina.

Sisci R., Lo Curzio M., Chillemi F. (1990). Messina, fortificazioni e Arsenali, strutture storiche e realtà urbana. EDAS ed. Messina. 